\title{
Nonlinear Stability Analysis of Transitional Flows using Quadratic Constraints
}

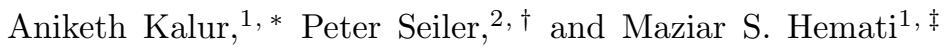 \\ ${ }^{1}$ University of Minnesota, Minneapolis, MN 55455 \\ ${ }^{2}$ University of Michigan, Ann Arbor, MI 48109
}

\begin{abstract}
The dynamics of transitional flows are governed by an interplay between the non-normal linear dynamics and quadratic nonlinearity in the incompressible Navier-Stokes equations. In this work, we propose a framework for nonlinear stability analysis that exploits the fact that nonlinear flow interactions are constrained by the physics encoded in the nonlinearity. In particular, we show that nonlinear stability analysis problems can be posed as convex feasibility and optimization problems based on Lyapunov matrix inequalities, and a set of quadratic constraints that represent the nonlinear flow physics. The proposed framework can be used to conduct global stability, local stability, and transient energy growth analysis. The approach is demonstrated on the low-dimensional Waleffe-Kim-Hamilton model of transition and sustained turbulence. Our analysis correctly determines the critical Reynolds number for global instability. For local stability analysis, we show that the framework can estimate the size of the region of attraction as well as the amplitude of the largest permissible perturbation such that all trajectories converge back to the equilibrium point. Additionally, we show that the framework can predict bounds on the maximum transient energy growth. Finally, we show that careful analysis of the multipliers used to enforce the quadratic constraints can be used to extract dominant nonlinear flow interactions that drive the dynamics and associated instabilities.
\end{abstract}

\section{INTRODUCTION}

Many complex flow phenomena arise from the interplay between the non-normal linear dynamics and quadratic nonlinearity in the incompressible Navier-Stokes equations (NSE). In wall-bounded shear flows, the high-degree of non-normality of the linearized NSE results in a transient energy growth (TEG) of small flow perturbations [1- $[3]$, even when the dynamics are linearly asymptotically stable. As a result, the linear analysis tends to over predict the critical Reynolds number $\left(R e_{c}\right)$ for instability in many shear flows [1, 2, 4. The fact that the flow transitions at Reynolds numbers $(R e)$ below the predicted linear stability limit is partly attributed to the non-modal growth that pushes the flow state away from the equilibrium base flow [1]. Indeed, TEG is a necessary condition for transition [5, 6]. Nevertheless, non-modal TEG alone is not sufficient to cause transition: it is the interaction of non-modal TEG with the nonlinearity that triggers secondary instabilities and drives the state outside the region of attraction. Without the nonlinear terms, the notion of a finite region of attraction would not make sense. Interestingly, although the nonlinearity is lossless and energy-conserving [5, 7, it can interact with the linear dynamics in such a way as to increase the maximum transient energy growth (MTEG) that can be realized 8 . These transition scenarios cannot be fully analyzed without accounting for the nonlinear terms in the NSE.

Analysis methods have been proposed to account for the interplay between the linear and nonlinear terms in transitional and turbulent flows. One such approach is the resolvent analysis framework [9 11, which leverages the fact that the NSE can be expressed as a feedback interconnection between a linear operator and a nonlinear operatora so-called Lur'e decomposition 12. Resolvent analysis goes a step further to consider the nonlinearity as an implicit forcing input on the linear dynamics [9, 13. This perspective greatly simplifies the resulting analysis problem, as only the linear system - described by the input-output properties of the linear resolvent operator - needs to be analyzed. Within the context of turbulent flows, resolvent analysis provides information on how fluctuations in a time-averaged flow are attenuated or amplified from nonlinear effects. Resolvent analysis has been successfully employed in the study of various flows [14, 15, including pipe flows [16, open cavity flows [17, and flows over riblets [18.

Related methods have been proposed to account for the nonlinearity in NSE more directly. The passivity framework has been shown to be effective in flow control based on the nonlinear NSE [19 21. In these studies, the passivity property [12] of the nonlinear terms in the incompressible NSE are leveraged to design a linear controller that can stabilize the system. Further advances have been made in input-output methods to study performance, worst-case amplification, stability, and transition for NSE using dissipation inequalities 22. Dissipation inequalities derived from NSE can be posed as linear matrix inequality (LMI) problems, which are then solved using convex optimization

\footnotetext{
* Graduate Student, Aerospace Engineering and Mechanics

$\dagger$ Associate Professor, Electrical Engineering and Computer Science

$¥$ Assistant Professor, Aerospace Engineering and Mechanics
} 
methods to analyze various wall-bounded shear flows. These techniques generalize the classical energy-based analysis approaches [1, 23. and also have close ties with nonlinear Lyapunov stability analysis approaches developed for NSE based on sum-of-squares (SOS) optimization [24.

In this paper, we propose an alternate framework for nonlinear stability analysis that uses quadratic constraints to account for nonlinear flow interactions with minimal complexity. The approach is predicated on the fact that nonlinear flow interactions are constrained by the physics encoded within the nonlinear terms in the incompressible NSEe.g., the nonlinearity is quadratic, energy conserving, and lossless. Mathematically, these physics can be expressed as quadratic constraints between the inputs and outputs of the nonlinearity. In turn, these quadratic constraints serve as reduced-complexity models for the nonlinear terms, and can be incorporated within a Lyapunov-based analysis to perform reliable stability and input-output analysis in the nonlinear setting. The general framework introduced here is applicable to any system that has (non-normal) linear dynamics acting in feedback with a lossless nonlinearity - the incompressible NSE being a special case.

To establish a proof-of-concept, we formulate and demonstrate the proposed analysis framework on the nonlinear Waleffe-Kim-Hamilton (WKH) model of transition and sustained turbulence [5]. As with the NSE, the WKH model admits a Lur'e decomposition with non-normal linear dynamics and a quadratic lossless nonlinearity, making it relevant for formulating and demonstrating the proposed quadratic constraints framework for nonlinear stability analysis of fluid flows.

The paper proceeds as follows. In Section II we introduce the WKH model in Lur'e form. We then introduce the quadratic constraints framework and associated stability analysis problem in Section III In Section IIIA, we account for the energy conserving nonlinearity in global stability analysis via the addition of a quadratic lossless constraint. In Section IIIB, we show that additional quadratic constraints can be introduced to conduct local stability analysis, which is needed when $R e>R e_{c}$ and the equilibrium point is no longer globally asymptotically stable. In Section IV] we formulate the problem for obtaining the size of the largest permissible perturbation such that the state trajectories remain in the region of attraction. In Section $\mathrm{V}$, we formulate an analysis problem to determine bounds on the MTEG that can be realized by the system dynamics. In Section VI, we show that we can obtain insights into dominating nonlinear flow interactions that underlie the dynamics by analyzing the multipliers used to enforce the constraints within the analysis framework. Finally, we provide concluding remarks of our study in Section VII]

The section-wise specific contributions of this paper are as follows:

1. Section IIIA We find that the lossless constraint alone enables prediction of the $R e_{c}$ for global instability, consistent with the $R e_{c}$ found by other means in [6].

2. Section IIIB and IIIC The "local" quadratic constraints represent the influence of the nonlinearity when the flow is restricted to a local neighborhood about the equilibrium point. We also show that the local region corresponds to the region of attraction and study how the region of attraction changes with Re.

3. Section IV] We show that the proposed framework can be used to predict the size of largest permissible perturbation such that system stability is ensured (i.e., a "transition threshold"). We find that the size of the permissible perturbations decreases with increasing $R e$.

4. Section $\mathrm{V}$ The proposed framework can be used to estimate bounds for MTEG in the nonlinear system. We verify the results from the proposed framework with nonlinear simulations, which indicate that the bounds can be conservative.

5. Section VI We demonstrate that - without any a priori knowledge - the proposed analysis approach is able to extract the same dominant nonlinear flow interactions whose physical importance is argued in [5, 6].

\section{WALEFFE-KIM-HAMILTON MODEL OF TRANSITION AND SUSTAINED TURBULENCE}

The Waleffe-Kim-Hamilton (WKH) model is a low-order mechanistic model for transition and sustained turbulence in shear flows. The model is based on observations from direct numerical simulations (DNS) of a plane Couette flow [25], and was introduced to highlight the importance of nonlinear interactions with the non-normal linear dynamics in the NSE. The WKH model was studied in greater detail by Waleffe in [6] and is given by,

$$
\left[\begin{array}{c}
\dot{u} \\
\dot{v} \\
\dot{w} \\
\dot{m}
\end{array}\right]=\frac{1}{R e}\left[\begin{array}{l}
0 \\
0 \\
0 \\
\sigma
\end{array}\right]-\frac{1}{R e}\left[\begin{array}{c}
\lambda u \\
\mu v \\
\nu w \\
\sigma m
\end{array}\right]+\left[\begin{array}{cccc}
0 & 0 & -\gamma w & v \\
0 & 0 & \delta w & 0 \\
\gamma w & -\delta w & 0 & 0 \\
-v & 0 & 0 & 0
\end{array}\right]\left[\begin{array}{c}
u \\
v \\
w \\
m
\end{array}\right] .
$$


Here, $R e$ denotes the Reynolds number; $u$ represents the amplitude of the spanwise modulation of streamwise velocity; $v$ represents the amplitude of the streamwise rolls; $w$ represents the amplitude of the inflectional streak instability; and $m$ represents the amplitude of the mean shear [6]. The constants $\lambda, \mu, \nu, \sigma$ are positive parameters corresponding to viscous decay rates. The constants $\gamma$ and $\delta$ represent nonlinear interaction coefficients and should have the same $\operatorname{sign}[6]$.

The WKH system in Eq. (1) captures the processes underlying sustained turbulence [6]: the rolls $(v)$ create streaks $(u)$ which eventually break down to maintain the rolls. In the WKH model, the mean shear $m$ is not frozen in time and changes due to nonlinear interactions captured by the $-v u$ term. A few important aspects of this model are that the instability $w$ grows from the streaks $u$ via the $\gamma w u$ interaction term. It can also be observed that the same instability feeds streamwise rolls by nonlinear quadratic interactions $\delta w^{2}$. The nonlinear couplings between $w$ and $v$ are important in sustaining turbulence [6]. As we will show in Section VI, the importance of these same interactions in driving instabilities can be identified from our proposed analysis framework.

The WKH model admits a laminar equilibrium point at $(u, v, w, m)_{e}=(0,0,0,1)$. For the proposed stability analysis, we perform a change of coordinates to translate the equilibrium point of Eq. (1) to the origin. The equilibrium point in these new coordinates is $\mathbf{x}_{e}=(0,0,0,0)$ and the state is $\mathbf{x}=(u, v, w, \bar{m})$, where $\bar{m}=m-1$. The system in this translated coordinate system is,

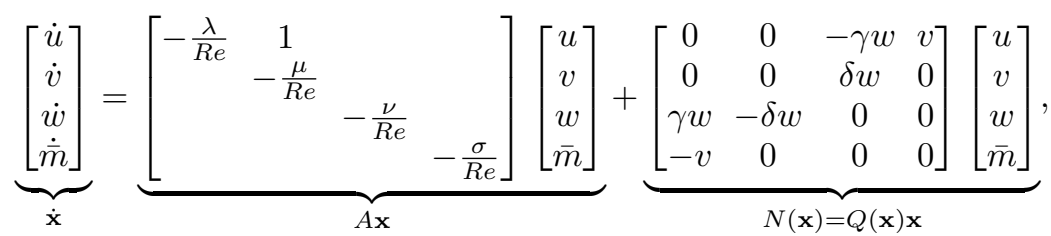

which makes the non-normality of the linear dynamics explicit [26].

The WKH system in Eq. (2) can be represented as

$$
\dot{\mathbf{x}}=A \mathbf{x}+N(\mathbf{x})
$$

where the linear operator $A$ is non-normal and asymptotically stable, and $N(\mathbf{x})$ is a quadratic nonlinearity given by $N(\mathbf{x})=Q(\mathbf{x}) \mathbf{x}$. Note that the nonlinear term is skew-symmetric: i.e., $Q(\mathbf{x})=-Q(\mathbf{x})^{T} \in \mathbb{R}^{4 \times 4}$. The linear and nonlinear terms can be partitioned into Lur'e form [12, with the two systems acting in feedback with each other (see FIG. 1]:

$$
\begin{aligned}
\dot{\mathbf{x}} & =L(\mathbf{x}, \mathbf{z}):=A \mathbf{x}+\mathbf{z} \\
\mathbf{z} & =N(\mathbf{x})
\end{aligned}
$$

where $\mathbf{z} \in \mathbb{R}^{4}$. This Lur'e decomposition of the WKH system is denoted as an upper linear fractional transformation $F_{u}(L, N)$. The advantage of writing this system in a Lur'e form is that Eq. (4a) alone is a linear dynamical system with an input $\mathbf{z}$. Although $\mathbf{z}$ is a nonlinear forcing given by Eq. 4b), we can instead account for it using input-output properties of $\mathbf{z}=N(\mathbf{x})$. In this way, we are able to perform a nonlinear analysis of WKH model through analysis of linear dynamics in Eq. (4a) subject to constraints between $\mathbf{x}$ and $\mathbf{z}$ determined by Eq. 4b.

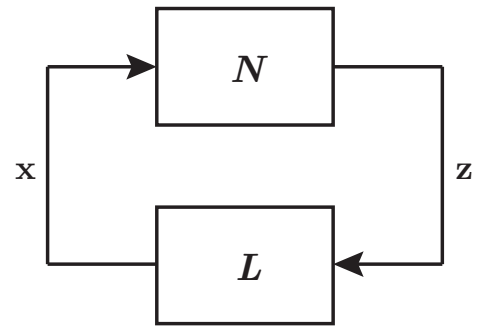

FIG. 1. Lur'e representation of the WKH system $F_{u}(L, N)$.

In all that follows, we study the proposed framework on the WKH system with two separate sets of parameters (see TABLE I). Both sets of parameters have been investigated in prior studies: the Waleffe (W) parameters in 6 , and the Baggett and Trefethen (B\&T) parameters in [27]. The W and B\&T parameters each yield different behaviors in the system dynamics, and the parameters chosen in this study result in notable differences in stability regions, 
permissible perturbation size, and transient energy growth 6, 27. In the remainder of this work, the only parameter that is varied for stability and transient energy growth analysis is Re. Other choices of parameters are possible, and changing of the nonlinear interaction coefficients will lead to different types of bifurcations and correspondingly different stability regimes.

\begin{tabular}{|c|c|c|c|c|c|c|}
\hline Parameter set & \multicolumn{5}{|c|}{ Parameter Value } \\
\hline \hline & $\lambda$ & $\mu$ & $\nu$ & $\sigma$ & $\delta$ & $\gamma$ \\
\hline W [6] & 10 & 10 & 15 & 10 & 1 & 0.1 \\
\hline B\&T [27] & 1 & 1 & 1 & 1 & 1 & 1 \\
\hline
\end{tabular}

TABLE I. The two parameter sets and the value of the corresponding coefficients used in this work.

\section{NONLINEAR STABILITY ANALYSIS USING QUADRATIC CONSTRAINTS}

Lyapunov stability methods [12 can be used to analyze the stability of a system given by Eq. (4). Here, the stability is analyzed around the equilibrium point $\mathbf{x}_{e}=\mathbf{0}$. To analyze stability using Lyapunov stability methods, we define a quadratic scalar energy function $V: \mathbb{R}^{n} \rightarrow \mathbb{R}$. The energy function $V(\mathbf{x})=\mathbf{x}^{T} P \mathbf{x}$ is a candidate Lyapunov function [12. From the Lyapunov stability theorem, the equilibrium point $\mathbf{x}_{e}=\mathbf{0}$ is globally asymptotically stable when $d V(\mathbf{x}) / d t<0 \forall \mathbf{x} \neq \mathbf{0}, t>0$ and $P \succ 0{ }^{1}$. In other words, the system is globally asymptotically stable around the equilibrium point $\mathbf{x}_{e}=\mathbf{0}$ if the energy continuously decreases in time. The time derivative of the Lyapunov function for the nonlinear system in Eq. (4), along trajectories of the system is given by:

$$
\begin{aligned}
\frac{d}{d t} V(\mathbf{x}) & =2 \mathbf{x}^{T} P(A \mathbf{x}+\mathbf{z}) \\
& =2 \mathbf{x}^{T} P(A \mathbf{x}+N(\mathbf{x})) .
\end{aligned}
$$

Including the effects of $\mathrm{N}(\mathbf{x})$ to analyze stability is crucial to understanding the global asymptotic stability of the nonlinear system. However, accounting for the nonlinear term $\mathrm{N}(\mathbf{x})$ complicates the stability analysis and a quadratic Lyapunov function will not necessarily be a good choice as a candidate Lyapunov function. Here, we leverage the fact that the inputs and outputs of the nonlinearity $N$ satisfy a set of quadratic constraints, thereby enabling stability analysis of the whole feedback interconnection $F_{u}(L, N)$ without the full complexity involved in an explicit treatment of the nonlinearity.

To do so, we first show that stability analysis benefits from consideration of the nonlinear term as energy conserving and lossless, neither producing nor dissipating energy. The lossless property can be represented as a quadratic constraint to represent the nonlinear term within the Lyapunov analysis. The lossless constraint captures global behavior of the nonlinearity. Further, we analyze local behavior of the nonlinearity around a neighborhood by representing its local properties as "local" constraints. We also show that local nonlinear properties play a role in destabilizing the system, whereas a linear stability analysis predicts the WKH system to be globally asymptotically stable for all Re. Both global and local stability analysis and the results are discussed in the following sections.

\section{A. Stability: Representing Lossless Nonlinearity with Quadratic Constraints}

The nonlinear term in Eq. 2) is skew-symmetric, therefore

$$
\mathbf{x}^{T} N(\mathbf{x})=\mathbf{x}^{T} Q(\mathbf{x}) \mathbf{x}=0, \forall \mathbf{x}
$$

The physical interpretation of this property is that the nonlinearity is energy conserving, serving only to redistribute energy between modes. This "lossless" property of the nonlinear term is also observed in many wall-bounded shear flows [7. The stability analysis reduces to the following question: Does the constraint in Eq. $(6)$ imply $\dot{V}(\mathbf{x})<0$ in Eq. (5) for all $\mathbf{x} \neq \mathbf{0}$ ? The answer is yes, if there exists a $P \succ 0$ and a Lagrange multiplier $\xi_{p_{0}}$ (positive or negative) such that

\footnotetext{
1 The condition " $\forall t>0$ " is implied for all Lyapunov-based arguments, even without explicit statement. Also, the relational operators $\prec 0, \succ 0(\preceq 0, \succeq 0)$ denote positive and negative (semi-) definite matrices, respectively.
} 


$$
2 \mathbf{x}^{T} P(A \mathbf{x}+N(\mathbf{x}))+2 \xi_{p o} \mathbf{x}^{T} N(\mathbf{x})<0,
$$

which essentially says that the energy function $V(\mathbf{x})$ decreases for any $\mathbf{x}$ and $N(\mathbf{x})$ satisfying the lossless constraint in Eq. (6).

Consider now that the lossless property in Eq. (6) can be expressed equivalently as a quadratic constraint between the inputs $\mathbf{x}$ and outputs $\mathbf{z}=N(\mathbf{x})$ of the nonlinearity:

$$
\left(\begin{array}{l}
\mathbf{x} \\
\mathbf{z}
\end{array}\right)^{T} \underbrace{\left(\begin{array}{ll}
\mathbf{0} & \mathbf{I} \\
\mathbf{I} & \mathbf{0}
\end{array}\right)}_{:=M_{0}}\left(\begin{array}{l}
\mathbf{x} \\
\mathbf{z}
\end{array}\right)=0, \forall \mathbf{x} \text { and } \mathbf{z} \in \mathbb{R}^{4},
$$

where $\mathbf{0}, \mathbf{I} \in \mathbb{R}^{4 \times 4}$ denote the zero and identity matrices, respectively. Thus, Eq. (7) can be recast as,

$$
\left[\begin{array}{l}
\mathbf{x} \\
\mathbf{z}
\end{array}\right]^{T}\left\{\left[\begin{array}{cc}
A^{T} P+P A & P \\
P & \mathbf{0}
\end{array}\right]+\xi_{p_{0}} M_{0}\right\}\left[\begin{array}{l}
\mathbf{x} \\
\mathbf{z}
\end{array}\right]<0 .
$$

The lossless constraint $\mathbf{z}^{T} \mathbf{x}=0$ is captured by the block matrix $M_{0} \in \mathbb{R}^{8 \times 8}$ defined in Eq. (8). The term in braces in Eq. (9) must be strictly negative definite for the strict inequality to be satisfied. However, for a matrix to be strictly negative definite, it is necessary for all principle sub-matrices to also be strictly negative definite [28, 29]. The bracketed term has $\mathbf{0}$ as a principle sub-matrix and hence the strict inequality in Eq. (9) cannot be satisfied. As such, we introduce a positive perturbation on Eq. (7) to relax the requirement for a strict inequality as,

$$
2 \mathbf{x}^{T} P(A \mathbf{x}+N(\mathbf{x}))+2 \xi_{p o} \mathbf{x}^{T} N(\mathbf{x})+2 \epsilon \mathbf{x}^{T} P \mathbf{x} \leq 0
$$

where $\epsilon>0$. This new condition is equivalent to $\dot{V}(\mathbf{x}) \leq-\epsilon V(\mathbf{x})$ for all $\mathbf{x} \neq \mathbf{0}$, which guarantees exponential stability with a minimum convergence rate of $\epsilon$ when satisfied.

The stability condition in Eq. (10) can be recast in terms of the quadratic lossless constraint in Eq. (8) to yield,

$$
\left[\begin{array}{l}
\mathbf{x} \\
\mathbf{z}
\end{array}\right]^{T}\left\{\left[\begin{array}{cc}
A^{T} P+P A & P \\
P & \mathbf{0}
\end{array}\right]+\xi_{p_{0}} M_{0}+\left[\begin{array}{cc}
\epsilon P & \mathbf{0} \\
\mathbf{0} & \mathbf{0}
\end{array}\right]\right\}\left[\begin{array}{l}
\mathbf{x} \\
\mathbf{z}
\end{array}\right] \leq 0
$$

Unlike the stability condition in Eq. (9), it is possible for this new stability condition in Eq. (11) to be satisfied because the inequality is non-strict. For Eq. (11) to hold, the matrix in braces has to be negative semi-definite. However, from the generalized Schur's complement 30 we know that the bracketed term will be negative semi-definite if and only if both $A^{T} P+P A+\epsilon P \preceq 0$ and $P+\xi_{p 0} \mathbf{I}=0$ for some $\xi_{p 0}<0$. Note that the condition $A^{T} P+P A+\epsilon P \preceq 0$ is the condition for verifying exponential stability of the linear system and by itself does not account for the nonlinearity; the addition of the quadratic constraints accounts for the lossless nonlinearity in this stability condition. In the limit $\epsilon \rightarrow 0$, this condition is equivalent to $A+A^{T} \prec 0$, which is a necessary and sufficient condition for unity maximum transient energy growth due to linear non-modal dynamics [31. Thus, our analysis is consistent with the fact that unity linear MTEG is necessary for global stability in the nonlinear system.

In light of the stability condition in (11), it follows that stability of the linear element $L$ and a lossless nonlinearity can be formulated as an LMI feasibility problem in the variables $P \succ 0$ and $\xi_{p 0}$. In particular, the system $F_{u}(L, N)$ is globally asymptotically stable if there exists $P \succ 0$ and $\xi_{p_{0}}$ such that the following LMI holds for a given $\epsilon>0$ :

$$
\left[\begin{array}{cc}
A^{T} P+P A & P \\
P & \mathbf{0}
\end{array}\right]+\xi_{p_{0}} M_{0}+\left[\begin{array}{cc}
\epsilon P & \mathbf{0} \\
\mathbf{0} & \mathbf{0}
\end{array}\right] \preceq 0 .
$$

The feasibility of the LMI in Eq. 12 is only sufficient to establish the global asymptotic stability of the WKH system, as it only relies on the lossless property and does not depend on any other specific details of the nonlinearity. The condition in Eq. 12 is an LMI feasibility problem that can be solved using standard numerical tools. Unless otherwise specified, in the remainder of this work, we use CVX [32, 33, which is a package for specifying convex optimizations, combined with the commercially available solver MOSEK 34 .

To analyze the global stability of the WKH system, we solve the LMI in Eq. 12 with $\epsilon=10^{-6}$ for variables $P$ and $\xi_{p_{0}}$ at different values of Re. On performing the global stability analysis using the lossless constraint, we find that the WKH model for the $\mathrm{W}$ parameter values (see TABLE I) is globally asymptotically stable for $R e \leq 20$. This finding is consistent with $R e_{c}=20$ for global asymptotic stability reported by Waleffe [6. Similarly we find that the WKH system with B\&T parameters (see TABLE【) is globally asymptotically stable for $R e \leq 2$. 


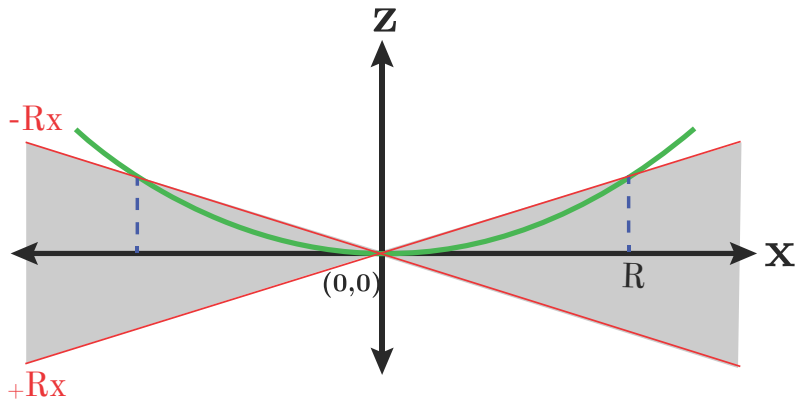

(a) Large slope $R$ forms larger sectors

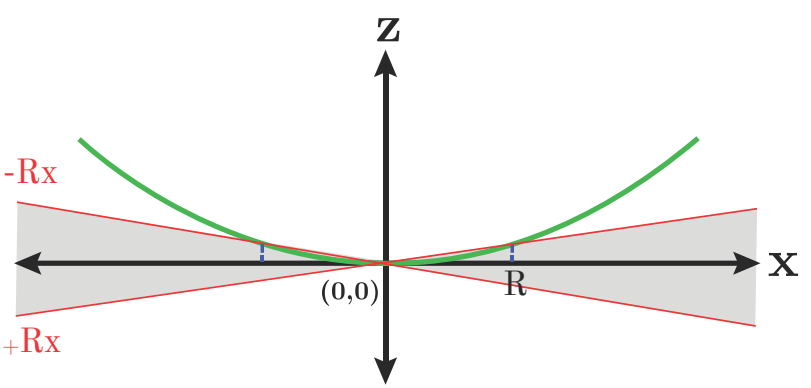

(b) Small slope $R$ forms smaller sectors

FIG. 2. Illustration of a scalar quadratic function $x=z^{2}$ that lies inside the sector formed by lines of slope $-R$ and $R$ (red). The blue dashed lines mark the maximum value of the pair $(x, z)$ for a given slope such that $|x| \leq R$.

Note that the linear WKH system is globally asymptotically stable for all $R e$, and so the nonlinear term is destabilizing. In the nonlinear WKH system, considering only the lossless constraint, we show that global stability cannot be established for $R e>20$ for the $\mathrm{W}$ parameters and $R e>2$ for the B\&T parameters. To investigate this further, we propose a set of local constraints on the nonlinearity that enable a local stability analysis, as described in the next section.

\section{B. Stability: Representing Local Properties of Nonlinearity using Quadratic Constraints}

The WKH system with the lossless constraint is globally asymptotically stable for $R e \leq 20$ for the W parameters and for $R e \leq 2$ for the B\&T parameters. To analyze the system for larger Re, we propose a "local" stability analysis as follows: Select a local neighborhood $\|\mathbf{x}\|^{2} \leq R^{2}$ around the equilibrium point $\mathbf{x}_{e}=\mathbf{0}$. Local analysis restricts the state $\mathbf{x}$ to lie in a local region $R$, which result in "local" constraints for $N(\mathbf{x})$ within this local region. The analysis condition, given below, attempts to use these local quadratic constraints to show that: (i) the system state remains within the local region and (ii) it converges asymptotically back to $\mathbf{x}_{e}=\mathbf{0}$. These quadratic constraints are tighter (more powerful) for smaller values of $R$ and become looser (less powerful) as $R$ becomes larger. Thus, these local analysis results provide a range of results between global asymptotic stability (roughly as $R \rightarrow \infty$ ) and stability of the linearized system (roughly as $R \rightarrow 0$ ). We will show later in this section that $R$ can be used to estimate the region of attraction (ROA) for the equilibrium point.

Recall that the nonlinearity in the WKH model is quadratic and can be expressed as $\mathbf{z}=\mathbf{x}^{T} Q(\mathbf{x}) \mathbf{x}($ see Eq. $(2)$ ). To illustrate the approach, first consider the scalar example $z=x^{2}$ (green curve in FIG. 2). Within a given region $|\mathbf{x}|<R$, the output satisfies $z^{2}=x^{4}<R^{2} x^{2}$. Which further implies that $|z|<R|x|$, where $R$ is the slope of the line. The quadratic function is restricted by the bound $\mathrm{R}$, but this bound would graphically correspond to drawing a line of slope $+R$ and $-R$ (red lines in FIG. 2). The slope $R$ can have a large value or a small value, as illustrated in FIGs. 2a and 2b, respectively. If $x$ remains in the interval $[-R,+R]$, then the nonlinear function lies between these two linear lines with slope $\pm R$ (gray shaded region in FIGs. 2a and 2b. The dashed blue line in both these figures represents the maximum possible value of the pair $(x, z)$, such that $|x| \leq R$ for a given slope. It can be seen that as the slope $R$ is made larger (FIG. 2a), then the pair $(x, z)$ also gets bigger, thereby moving the blue dashed line further away from the origin. Similarly, as slope of $R$ is made smaller (FIG. $2 \mathrm{~b}$ ), the pair $(x, z)$ gets smaller, thereby moving the dashed line towards the origin, which corresponds to a reduction in maximum value of $z$. Finally, note that as the slope $R$ tends to zero, the sector shrinks to zero. Thus, $R \rightarrow 0$ corresponds to a nonlinear term with zero output - equivalent to a linear analysis. Conversely, as $R \rightarrow \infty$, then this sector becomes arbitrarily large and provides essentially no information - corresponding to a global analysis.

The sector formed by lines of slope $\pm R$ facilitates bounding the pair $(x, z)$ to perform analysis in a localized setting, where the value of $R$ also determines the amount of nonlinear behavior captured by the local constraint. A brief introduction to scalar sector bounded nonlinearities is presented in Appendix A. The remainder of this section generalizes this basic concept to the multivariable quadratic terms that appear in the WKH model.

From Eq. (2), each individual nonlinear term can be expressed as a quadratic function: 


$$
\mathbf{z}=\left[\begin{array}{c}
z_{1} \\
z_{2} \\
z_{3} \\
z_{4}
\end{array}\right]=\left[\begin{array}{c}
\mathbf{x}^{T} Q_{1} \mathbf{x} \\
\mathbf{x}^{T} Q_{2} \mathbf{x} \\
\mathbf{x}^{T} Q_{3} \mathbf{x} \\
\mathbf{x}^{T} Q_{4} \mathbf{x}
\end{array}\right]
$$

Here each $Q_{i} \in \mathbb{R}^{4 \times 4}$ is a symmetric matrix. Hence each $Q_{i}$ has real eigenvalues, and the spectral radius $\rho\left(Q_{i}\right)$ denotes the largest (magnitude) of these eigenvalues [29. Moreover, quadratic terms with symmetric matrices are upper bounded as follows [29]:

$$
\left|z_{i}\right|=\left|\mathbf{x}^{T} Q_{i} \mathbf{x}\right| \leq \rho\left(Q_{i}\right) \mathbf{x}^{T} \mathbf{x}, \text { for } i=1 \text { to } 4 .
$$

Next, assume the state $\mathbf{x}$ remains within a ball of radius $R$, i.e. $\mathbf{x}^{T} \mathbf{x} \leq R^{2}$. We can then square Eq. (14) to obtain the following constraint:

$$
z_{i}^{2} \leq \underbrace{\rho\left(Q_{i}\right)^{2} R^{2}}_{\alpha_{i}(R)^{2}} \mathbf{x}^{T} \mathbf{x}, \text { for } i=1 \text { to } 4 .
$$

This is a constraint involving squares of $\mathbf{x}$ and $z_{i}$. It can be written in a more useful quadratic constraint form. Let $E_{i} \in \mathbb{R}^{4 \times 4}$ denote the matrix with the diagonal $(i, i)$ entry equal to one and all other entries equal to zero. The constraint in Eq. 15 is equivalent to:

$$
\left[\begin{array}{l}
\mathbf{x} \\
\mathbf{z}
\end{array}\right]^{T} \underbrace{\left[\begin{array}{cc}
\alpha_{i}(R)^{2} \mathbf{I} & \mathbf{0} \\
\mathbf{0} & -E_{i}
\end{array}\right]}_{M_{i}(R)}\left[\begin{array}{l}
\mathbf{x} \\
\mathbf{z}
\end{array}\right] \geq 0, \text { for } i=1 \text { to } 4 .
$$

The above multivariable quadratic constraint in Eq. 16 is similar to the sector constraint in the scalar case, shown in Eq. A1 of Appendix A. The above constraint provides a bound on the nonlinear term $z_{i}$ that holds over the local region $\mathbf{x}^{T} \mathbf{x} \leq R^{2}$. A local bound can be obtained for each of the four quadratic nonlinearities in Eq. (16). It should be noted that the lower right block in each $M_{i}(R)$ matrix is non-zero, and so we can use the strict inequality $\dot{V}(\mathbf{x})+\xi_{p 0} M_{0}+\sum_{i=0}^{4} \xi_{p i} M_{i}<0$. We will make use of these local constraints to study local stability of the WKH system in section IIIC, and show that they can be used for transient energy growth analysis as well in Section VB.

\section{Results: Local stability analysis using quadratic constraints}

The lossless property in Eq. (8) captures the global behavior of the quadratic nonlinearity. Given that the WKH system is not globally stable for $R e>20$, it is still beneficial to understand its local stability properties. The linearization around $\mathbf{x}_{e}=\mathbf{0}$ is stable for all $R e>0$ because $A$ is Hurwitz. A more quantitative local stability analysis can be performed around $\mathbf{x}_{e}=\mathbf{0}$ using the local constraints derived in Eq. (16). Specifically, our goal is to estimate the region of attraction (ROA), which corresponds to the set of initial conditions whose trajectories converge back to $\mathbf{x}_{e}=0$. We will consider the local constraints on the nonlinearity that hold over the sphere $\|\mathbf{x}\| \leq R$. The local stability analysis for the nonlinear system can be performed by solving the following LMI feasibility problem:

$$
\begin{aligned}
& P \succeq I \\
& \xi_{p_{i} \geq 0} \quad(\text { for } i=1 \text { to } 4) \\
& {\left[\begin{array}{cr}
A^{T} P+P A & P \\
P & \mathbf{0}
\end{array}\right]+\xi_{p_{0}} M_{0}+\sum_{i=1}^{4} \xi_{p_{i}} M_{i}(R) \prec 0 .}
\end{aligned}
$$

Note that the local quadratic constraints depend on the radius $R$ as explicitly denoted by $M_{i}(R)$. If Eq. (17) has a feasible solution, then $V(\mathbf{x})=\mathbf{x}^{T} P \mathbf{x}$ is a Lyapunov function. Moreover, the level set $S_{R}:=\left\{\mathbf{x}: V(\mathbf{x})<R^{2}\right\}$ is an inner approximation to the region of attraction (ROA). These facts are formally proved in Appendix $B$. For a given $R e$ and $R$, we solve the feasibility problem in Eq. (17). If the problem is feasible for a region of size $R$, we know that the problem is also feasible for a region whose size is smaller than $R$ as well. This enables us to use bisection to obtain 
the lower bound on $R$. However, solving the feasibility problem via bisection for numerous $R$ can be computationally cumbersome. Hence, we re-pose the problem for finding the largest lower bound on $R$ as a quasi-convex generalized eigenvalue problem (GEVP) 35].

To find the estimate of the largest inner approximation for the ROA, we first decompose each local constraint matrix $M_{i}(R)$ as

$$
M_{i}(R)=R^{2} \underbrace{\left[\begin{array}{cc}
\rho\left(Q_{i}\right)^{2} & \mathbf{0} \\
\mathbf{0} & \mathbf{0}
\end{array}\right]}_{\tilde{M}_{i}}+\underbrace{\left[\begin{array}{cc}
\mathbf{0} & \mathbf{0} \\
\mathbf{0} & -E_{i}
\end{array}\right]}_{\hat{M}_{i}} .
$$

Now an estimate for the ROA can be obtained by using a change of variables $t=-R^{2}$ and solving the GEVP,

$$
\begin{aligned}
\operatorname{minimize} & t \\
\text { subject to } & P \succeq \mathrm{I} \\
& \xi_{p_{i}} \geq 0 \quad(\text { for } i=1 \text { to } 4) \\
& {\left[\begin{array}{cr}
A^{T} P+P A & P \\
P & \mathbf{0}
\end{array}\right]+\xi_{p_{0}} M_{0}+\sum_{i=1}^{4} \xi_{p_{i}} \hat{M}_{i} \prec t \sum_{i=1}^{4} \xi_{p_{i}} \tilde{M}_{i}, }
\end{aligned}
$$

where $\xi_{p_{i}}(i=1$ to 4$)$ are Lagrange multipliers for the local constraints. These Lagrange multipliers also provide information on the relative contribution of each constraint in the local region, as will be discussed in Section VI. In this work, the GEVPs are solved using LMI-Lab [36].

The analysis condition in Eq. (19) can be used to estimate the largest lower bound of $R$ as a function of $R e$. The resulting relationship between $R e$ and $R$ is shown in FIG. 3. Note that $R$ decreases monotonically as $R e$ tends to $\infty$. This implies that the local stability region shrinks as $R e$ increases. On the other hand, $R$ tends to $\infty$ as $R e$ decreases to 20 as shown in FIG. 3a for the W parameter case. In the $\mathrm{W}$ parameter case, the local stability region increases in size as $R e \rightarrow 20$. This is consistent with the previous global stability result, where $R e \leq 20$ was found to be global stable using only the lossless constraint.

Similarly, we also analyze the local stability for the B\&T parameters in FIG. 3b. Here, the system is globally stable for $R e \leq 2$, demarcated by the dashed red line in FIG. $3 \mathrm{~b}$. The same relation between $R$ and $R e$ is observed with the B\&T parameter as with $\mathrm{W}$ parameters; that is, the size of $R$ is decreases with increasing $R e$.

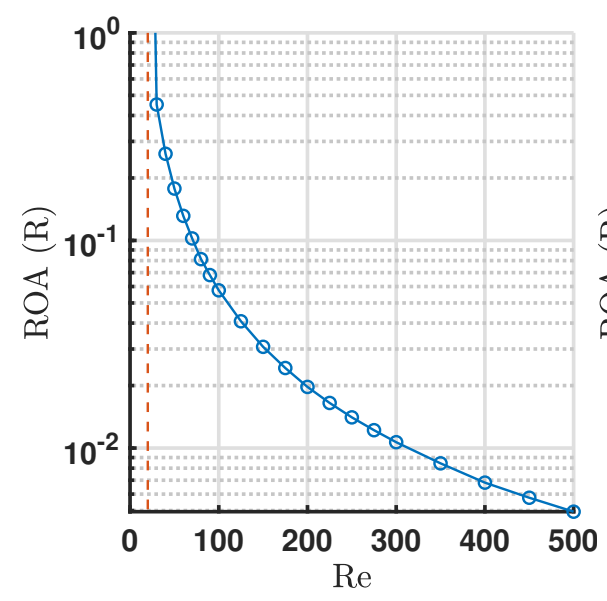

(a) W parameter

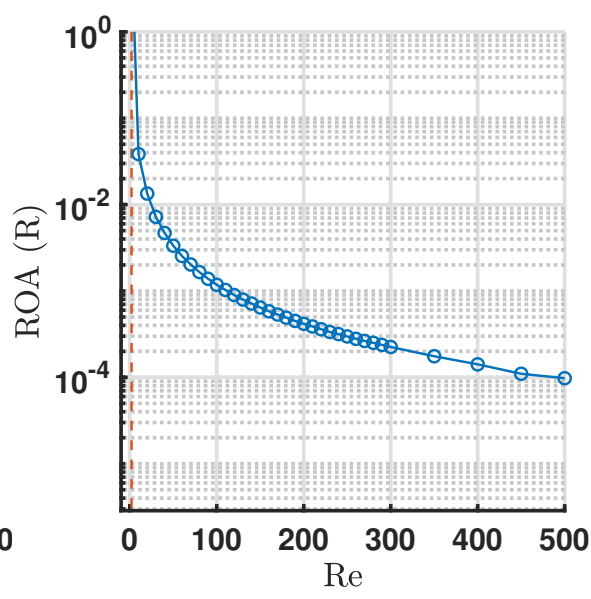

(b) B\&T parameter

FIG. 3. As the $R e$ is increased, the local stability region $R$ decreases. The red dashed line shows the Re for global stability limit. In both (a) and (b) as we approach the global stability limit, the size of $R \rightarrow \infty$. As $R e \rightarrow \infty$, the size of region $R \rightarrow 0$, which corresponds to the linear analysis of infinitesimal perturbations. 


\section{ESTIMATING PERMISSIBLE PERTURBATION AMPLITUDES}

We have shown that the GEVP in Eq. 119 can be solved to obtain the largest lower bound on $R$. Now we aim to identify the largest perturbation size $R_{0}$, such that trajectories originating in a sphere of radius $R_{0}$ will converge back to the equilibrium. The sphere of radius $R_{0}$ is obtained by finding the largest inner approximation of the ROA - this sphere is a sub-level set of the ellipsoid $V(\mathbf{x}) \leq R^{2}$. The size and shape of the ellipsoid depends on the energy weight matrix $P$ found in Eq. (19). We will show that the GEVP can be used to estimate the largest perturbation amplitude $R_{0}$ that drives the trajectory to the edge of stability.

Consider initial conditions inside a sphere of radius $R_{0}$ around the equilibrium point such that $\left\|\mathbf{x}_{0}\right\|^{2} \leq R_{0}^{2}$. Let $q:=\lambda_{\max }(P) / \lambda_{\min }(P)$ be the condition number of $P$ with $\lambda_{\max }$ and $\lambda_{\min }$ being the largest and smallest eigenvalues of $P$, respectively. The condition number $q$ of matrix $P$ provides information on the skewness of the bounding ellipsoid. To bound the skewness of the elliptical set, we can simply bound $P$ as $I \preceq P \preceq q I$. We now seek to determine the largest sphere of initial conditions $\left\|\mathbf{x}_{0}\right\|^{2} \leq R_{0}^{2}$ such that trajectories remain inside the ROA. From the inequality $I \preceq P \preceq q I$ and $\dot{V}(\mathbf{x})<0$, it follows that $\|\mathbf{x}\|^{2} \leq \mathbf{x}^{T} P \mathbf{x} \leq \mathbf{x}_{0}^{T} P \mathbf{x}_{0} \leq q\left\|\mathbf{x}_{0}\right\|^{2}$, which implies that $\|\mathbf{x}\|^{2} \leq q\left\|\mathbf{x}_{0}\right\|^{2}$. Therefore, we have $\|\mathbf{x}\|^{2} \leq q R_{0}^{2}=R^{2}$, and we can find the largest allowable sphere of radius $R_{0}$. To do so, we can now use the decomposition of $M_{i}=R^{2} \tilde{M}_{i}+\hat{M}_{i}$ (see Eq. (18) ) and the relation $R^{2}=q R_{0}^{2}$, then solve the following GEVP

$$
\begin{aligned}
\operatorname{minimize} & t_{0} \\
\text { subject to } & I \preceq P \preceq q I \\
& \xi_{p_{i}} \geq 0 \quad(\text { for } \mathrm{i}=1 \text { to } 4) \\
& {\left[\begin{array}{cr}
A^{T} P+P A & P \\
P & \mathbf{0}
\end{array}\right]+\xi_{p_{0}} M_{0}+\sum_{i=1}^{4} \xi_{p_{i}} \hat{M}_{i} \prec t_{0} q \sum_{i=1}^{4} \xi_{p_{i}} \tilde{M}_{i}, }
\end{aligned}
$$

where $t_{0}=-R_{0}^{2}$. To solve for the largest permissible perturbation amplitude, we solve Eq. 20) over a grid of $q$ values, then pick the solution corresponding to the largest $R_{0}$.

We verify our findings by comparing the $R_{0}$ obtained from the proposed quadratic constraints $(\mathrm{QC})$ framework with the nonlinear optimal perturbations obtained using nonlinear direct-adjoint looping (DAL), as detailed in [8, 37. In the DAL method, a so-called "minimal seed" is obtained using the calculus of variations and a gradient method to find the nonlinear optimal perturbation on a sphere of radius $R_{0}$. If divergent trajectories are found for perturbations of size $R_{0}$, we update the upper and lower bounds on $R_{0}$ via bisection. Once the upper and lower bounds converge to some tolerance $\epsilon>0$ (here $\epsilon=10^{-8}$ ) and no divergent trajectories are found, we terminate the bisection procedure. Since DAL uses the exact nonlinear equations of motion, it provides accurate estimates for the minimal seed of the nonlinear system and the permissible amplitude. In addition to the DAL method, we also compare with results from the sum-of-squares (SOS) framework using quadratic energy functions 24. Both the SOS and QC framework use Lyapunov-based methods to compute the largest sphere of radius $R_{0}$, which is an inner approximation of the ROA, and hence these methods are conservative in their estimates of the permissible perturbation amplitude.

In FIG. 4, we report the largest radius of initial conditions $R_{0}^{*}$ versus $R e$ using the three methods mentioned above. For the B\&T parameters we show results of the DAL $(\triangle)$, SOS framework $(\square)$, and QC framework $(\bigcirc)$ in FIG. $4 \mathrm{~b}$. However, for the W parameters we compare the results of the SOS framework and QC framework only in FIG. 4a, this is because for the W parameters no divergent trajectories were found using DAL. Even though there are no divergent trajectories the SOS framework and QC framework provide conservative estimates for $R_{0}$, as expected.

For the $\mathrm{B} \& \mathrm{~T}$ parameters, the largest allowable perturbation size for $R e=100$ is found to be $R_{0}^{*} \approx 1.5 \times 10^{-5}$ using the QC framework; this is a conservative estimate relative to $R_{0}^{*}=10^{-4}$ in [27. As seen in both figures in FIG. 4. we observe that the $R_{0}^{*}$ predicted by the QC framework is conservative. Since the QC framework does not use detailed information of the nonlinear terms - it only uses a few constraints that characterize the input-output behavior of the nonlinear terms - this behavior is expected. In exchange for this conservatism, the QC framework is less computationally expensive compared to the SOS and DAL methods, which are more computationally expensive and may not be suitable for large systems. We finally note that recent works by Liu and Gayme [38] - which appeared during the review of this manuscript - have aimed to reduce conservatism of these estimates within the QC framework.

\section{NONLINEAR TRANSIENT ENERGY GROWTH ANALYSIS USING QUADRATIC CONSTRAINTS}

In this section, we aim to determine the least upper bound on the maximum transient energy growth (MTEG) in the nonlinear system using the QC framework. We first review how the MTEG bounds can be obtained for a linear 


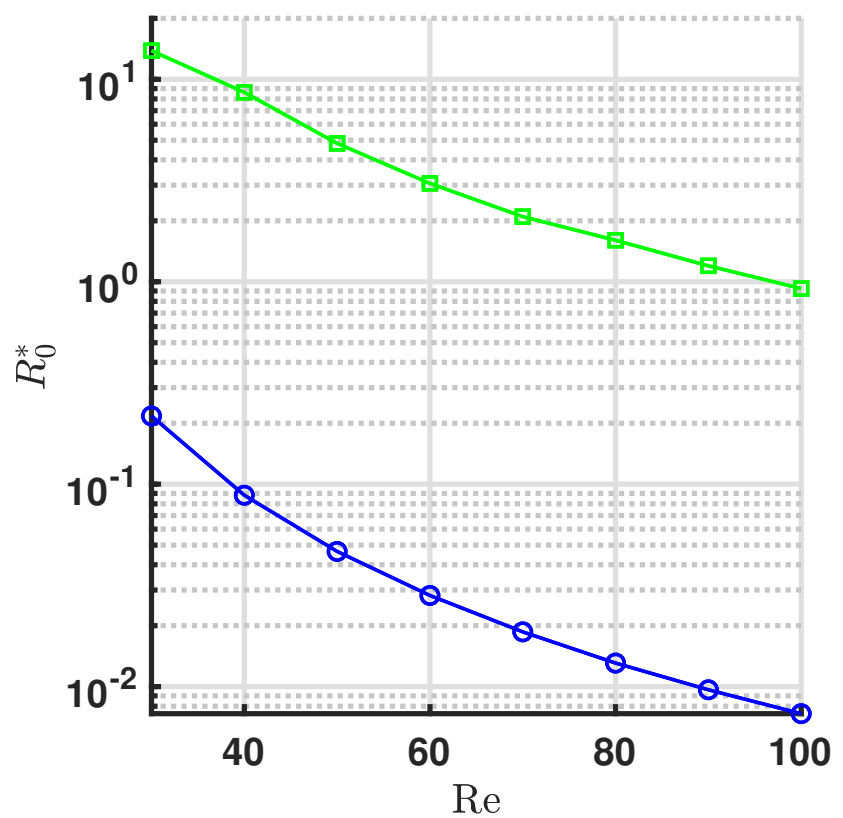

(a) W parameters

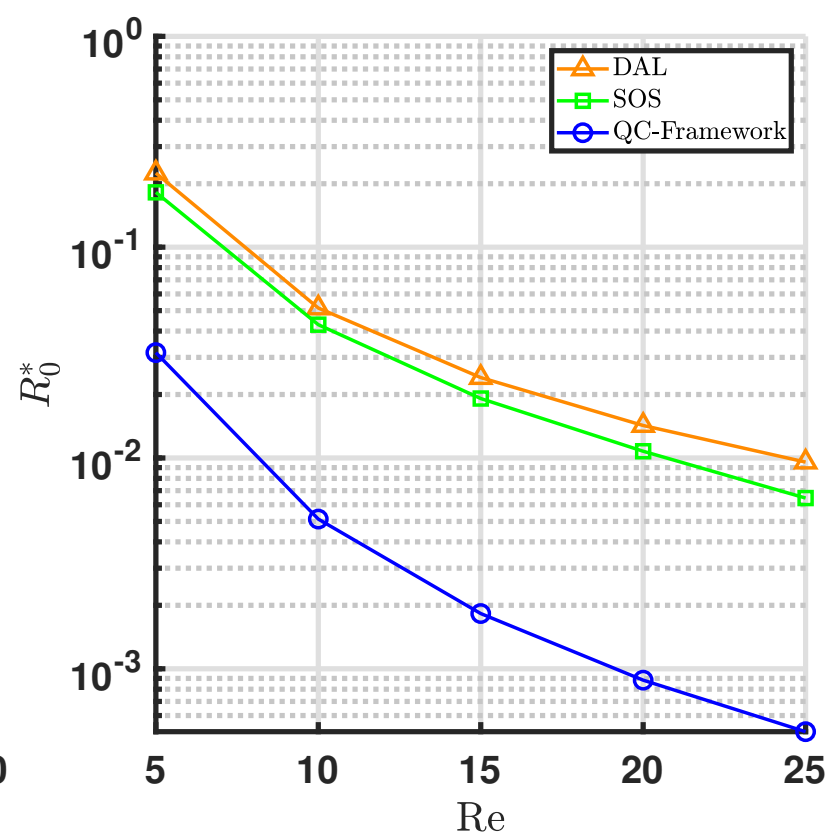

(b) B\&T parameters

FIG. 4. The maximum allowable perturbation size $R_{0}$ as a function of $R e$ for $\mathrm{W}$ and B\&T parameters compared against the SOS framework and DAL method.

system by solving a GEVP. We then extend this GEVP to determine the MTEG in the nonlinear system using the QC framework.

For a globally asymptotically stable linear system $\dot{\mathbf{x}}=A \mathbf{x}$, the state trajectories $\mathbf{x}(t) \rightarrow \mathbf{0}$ for any initial condition. If the matrix $A$ is non-normal, then the system energy $E:=\mathbf{x}^{T} \mathbf{x}$ will grow on transient time scales before decaying back to zero. The definition of energy $E$ here is without loss of generality, since any energy of the form $E=\tilde{\mathbf{x}}^{T} W \tilde{\mathbf{x}}$ is recovered with $\mathbf{x}=W^{1 / 2} \tilde{\mathbf{x}}$. In what follows, the associated MTEG, given by $\Theta$, is defined as:

$$
\Theta:=\max _{t \geq 0} \max _{\|\mathbf{x}(0)\|=1}\|\mathbf{x}(t)\|^{2}
$$

We can obtain an upper bound $q$ on the MTEG $(\Theta)$ using Lyapunov theory. Specifically, assume $V(\mathbf{x})=\mathbf{x}^{T} P \mathbf{x}$ is a Lyapunov function for a given system with $I \preceq P \preceq q I$. If the initial condition $\mathbf{x}_{0}$ lies in the ellipsoid $\left\{\mathbf{x}: \mathbf{x}^{T} P \mathbf{x} \leq 1\right\}$, then $\mathbf{x}(t)$ remains in this level set for all $t \geq 0$ [31, 39]. Thus $\mathbf{x}(t)^{T} P \mathbf{x}(t) \leq \mathbf{x}(0)^{T} P \mathbf{x}(0)$. Combining this with $I \preceq P \preceq q I$ yields $E(t) \leq \mathbf{x}(t)^{T} P \mathbf{x}(t) \leq \mathbf{x}(0)^{T} P \mathbf{x}(0) \leq q E(0)$. Therefore, $\Theta \leq q$ [40.

The least upper bound $q^{*}$ is defined as $q^{*}:=\lambda_{\max }(P) \lambda_{\max }\left(P^{-1}\right)$ such that $P=P^{T} \succ 0$ and $P$ satisfies $A^{T} P+P A \prec$ 0 . The problem of estimating $q^{*}$ can be formulated as that of minimizing the condition number of $P$ and is obtained by solving the following GEVP [31, 41]:

$$
\begin{aligned}
q^{*}:=\text { minimize } & q \\
\text { subject to } \mathrm{I} \preceq P \preceq q \mathrm{I}, & \\
& A^{T} P+P A \prec 0 .
\end{aligned}
$$

Here, the bounds $I \preceq P \preceq q I$ ensure that $\lambda_{\max }(P) / \lambda_{\min }(P) \leq q$. This optimization with LMI constraints and a linear cost involving variables $(P, q)$ is known as a semidefinite program (SDP). The LMI constraints imply that $V(\mathbf{x}):=\mathbf{x}^{T} P \mathbf{x}$ is a Lyapunov function for the system such that $V(\mathbf{x}(t)) \leq V(\mathbf{x}(0))$ for all $t \geq 0$. The bounds on $P$ further imply that $\|\mathbf{x}(t)\|^{2} \leq q^{*}\|\mathbf{x}(0)\|^{2}$. These LMI constraints are conservative in general, and hence $q^{*}$ is a (possibly non-tight) upper bound on the MTEG. Next, we will extend these ideas for MTEG analysis of nonlinear systems using QCs. 


\section{A. Global MTEG Analysis using Quadratic Constraints}

An optimization problem similar to Eq. 22 can be formulated to study the MTEG in the nonlinear WKH system. The lossless property for the nonlinear term in Eq. 8) can again be used as a global constraint. Taking a similar approach as in Section III A, we perturb the Lyapunov inequality to ensure a feasible solution can exist when only the lossless constraint is used. This yields the following optimization for a given $\epsilon>0$ :

$$
\begin{aligned}
q^{*}:=\operatorname{minimize} & q \\
\text { subject to } \mathrm{I} \preceq P \preceq q \mathrm{I} & \\
& {\left[\begin{array}{cc}
A^{T} P+P A & P \\
P & \mathbf{0}
\end{array}\right]+\xi_{p_{0}} M_{0}+\left[\begin{array}{cc}
\epsilon P & \mathbf{0} \\
\mathbf{0} & \mathbf{0}
\end{array}\right] \preceq 0 }
\end{aligned}
$$

Equation (23) is now a SDP in the variables $\left(P, q, \xi_{p_{0}}\right)$. As before, the LMI constraints imply that the Lyapunov function evaluated at the initial time upper bounds the Lyapunov function for all $t \geq 0$. The bounds on $P$ imply that $E(t) \leq q^{*} E(0)$ and are obtained by means of a Lyapunov function that describes a trajectory bounding ellipsoid, known as an invariant set. As with the linear MTEG analysis in Eq. 22, the quadratic constraint analysis problem in Eq. 23) is expected to yield a conservative upper bound on MTEG.

\section{B. Local MTEG Analysis using Quadratic Constraints}

The ability to obtain MTEG bounds is of interest even beyond the globally stable regime considered in Section $\mathrm{V}$. Hence, we use the local properties of the nonlinearity derived in Section III C to study the "local" MTEG performance in the nonlinear system.

A formulation similar to Eq. 23) can be used to study the effect of nonlinearity on MTEG in the nonlinear system. To perform the local MTEG analysis, additional local constraints are added to the optimization problem listed in Eq. (23). The local constraints that capture input-output properties of the nonlinear term are captured by matrices $M_{i}$ (for $i=1$ to 4 ) defined in Eq. (18). The addition of these constraints facilitates the study of local MTEG on the nonlinear system. The local MTEG for the nonlinear system is computed via the following convex optimization:

$$
\begin{aligned}
\operatorname{minimize} & q \\
\text { subject to } & \mathrm{I} \preceq P \preceq q \mathrm{I}, \\
& \xi_{p_{i}} \geq 0 \quad(\text { for } i=1 \text { to } 4) \\
& {\left[\begin{array}{cr}
A^{T} P+P A & P \\
P & \mathbf{0}
\end{array}\right]+\xi_{p_{0}} M_{0}+\sum_{i=1}^{4} \xi_{p_{i}} M_{i} \prec 0 . }
\end{aligned}
$$

We will identify MTEG bounds for the system about a local equilibrium point $\mathbf{x}_{e}=\mathbf{0}$ by solving this optimization for $P, q, \xi_{p_{0}}$, and $\xi_{p_{i}}$ (for $i=1$ to 4 ). The proof in Appendix B also applies for Eq. (24), therefore ensuring the states always remain inside the invariant set for all time $t \geq 0$.

\section{Results: MTEG analysis using quadratic constraints}

\section{Global MTEG analysis}

By solving the SDP in Eq. 23) with $\epsilon=10^{-6}$, we find that the MTEG bound is unity for all $R e<20$ using the W parameters. It is interesting to note that the linear part of the WKH system exhibits unity MTEG for $R e<20$ as well. Yet, the same MTEG bound from Eq. (23) is stronger because it applies to the nonlinear system $F_{u}(L, N)$ with a lossless nonlinearity. Similarly, from global MTEG analysis of the WKH system with B\&T parameters, we find that the system is globally stable for $R e \leq 2$ and the system holds unity MTEG for $R e<2$. 


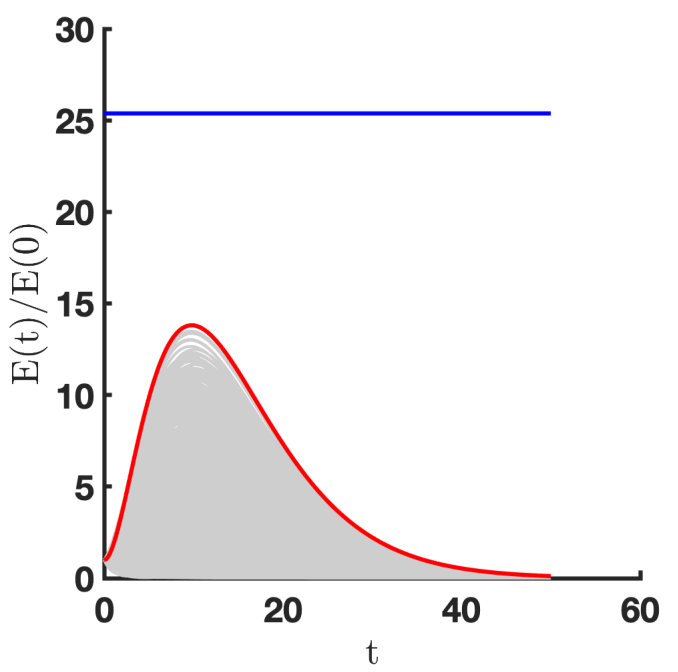

(a) $\mathrm{W}$ parameter, $R e=100$

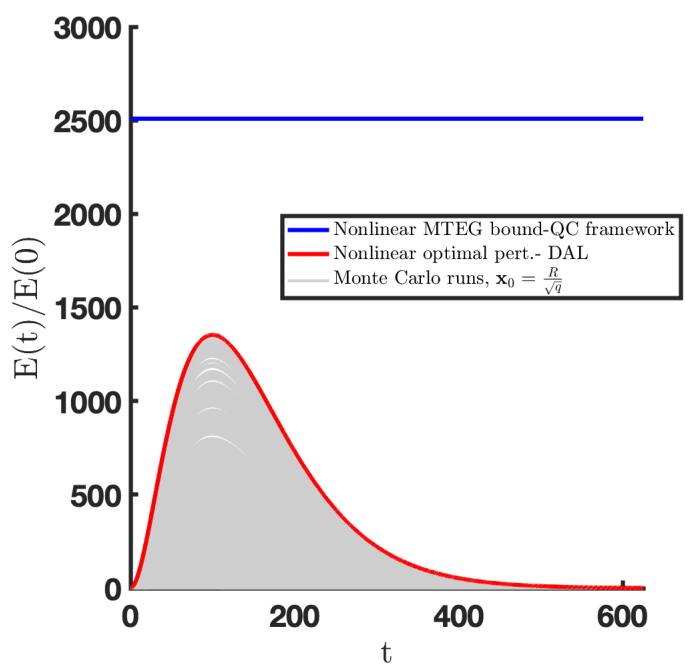

(b) B\&T parameter, $R e=100$

FIG. 5. 5000 Monte Carlo simulations from various initial conditions such that $\left\|\mathbf{x}_{0}\right\|^{2} \leq \frac{R}{\sqrt{q}}$, with $R=10^{-2}$ and $R=10^{-4}$ for W parameter and B\&T parameters respectively. The solid blue line shows the MTEG upper bound for nonlinear system predicted by the quadratic constraint framework proposed here. The solid gray curves show the Monte Carlo simulations for various initial conditions and the red curve shows the worst case MTEG of the nonlinear system.

For a given Re and local region $R$, we solve Eq. 24) for the MTEG bounds for both the W and B\&T parameters. In FIG. 5. we compare MTEG bounds from the QC framework with Monte Carlo simulations and the MTEG obtained from the DAL method 8, 37. From FIGs. 5a and 5b we see that MTEG bounds obtained from Eq. (24) (solid blue lines) are conservative. In FIG. 5, the solid gray curves correspond to the TEG from random initial conditions sampled with $\left\|\mathbf{x}_{0}\right\|=\frac{R}{\sqrt{q}}$, and the red curves correspond to MTEG resulting from the nonlinear optimal perturbation obtained using DAL. For the W parameters and the B\&T parameters, the MTEG bounds are reported for $R=10^{-2}$ and $R=10^{-4}$, respectively. The initial condition for the Monte Carlo simulations are obtained using the same $R$ value and the corresponding $q$ obtained from solving Eq. (24). Even though the QC framework provides a conservative estimate on the MTEG bounds, it can be an effective tool in analyzing transient energy growth in more complex system where DAL and Monte Carlo based estimates are computationally cumbersome, or in situations where exact information about the nonlinear terms may not be precisely known.

\section{LAGRANGE MULTIPLIER ANALYSIS: DRAWING PHYSICAL INSIGHTS INTO NONLINEAR FLOW INTERACTIONS}

In addition to providing a framework to analyze stability and transient energy growth, the quadratic-constraintsbased methods can be used to gain insights into the physics and dominating mechanisms underlying these dynamics. These insights are obtained by analyzing the Lagrange multipliers obtained from solving the GEVP in Eq. (20) as well as the SDP convex optimization problem in Eq. 24). The Lagrange multipliers provide information on the marginal cost of violating the associated constraints, thus indicating the relative importance of the corresponding constraints in the optimization problem. This allows for a quantitative analysis that can be used for identifying dominant nonlinear interactions. Here, we analyze the Lagrange multipliers for the $\mathrm{W}$ parameters, since Waleffe discusses these nonlinear interaction terms in 6], providing a basis for comparison. However, the same conclusions are obtained from the Lagrange multiplier analysis of the B\&T parameters as well.

The Lagrange multipliers obtained from solving Eq. 20) for $R_{0}$ with the $\mathrm{W}$ parameters over various $R e$ are shown in FIG. 6. In FIG. 6a we observe that the importance of the nonlinear terms varies as $R e$ is varied. In the initial phase for $R e<175$, we see the dominating Lagrange multipliers are $\xi_{p_{1}}$ and $\xi_{p_{2}}$ corresponding to nonlinear interaction terms $-\gamma w^{2}+v \bar{m}$ and $\delta w^{2}$, respectively. As Re increases, we see that the Lagrange multipliers $\xi_{p_{2}}$ (corresponding to $\left.\delta w^{2}\right)$ and $\xi_{p_{3}}$ (corresponding to $\gamma w u-\delta w v$ ) become more dominant with respect to the other multipliers. In FIG. 6a. it can be seen that $\xi_{p_{2}}$ is approximately 100 times more dominant than $\xi_{p_{3}}$ for $R e>200$. Further, $\xi_{p 3}$ is orders of magnitude larger than the multipliers associated with the other nonlinearities. Over all $R e$, the most 
dominant Lagrange multiplier is $\xi_{p_{2}}$ (i.e., the nonlinear term $\delta w^{2}$ ), while the least dominating Lagrange multiplier is $\xi_{p_{4}}$ (i.e., nonlinear interaction $-v u$ ). Inspecting Lagrange multipliers alone may not provide the complete picture, as the Lagrange multipliers can be influenced by the scaling of the constraint matrix $M_{i}$. Hence, we also plot the singular values of $\xi_{p_{i}} M_{i}$ - denoted by $\left.\sigma_{\max }\left(\xi_{p_{i}} M_{i}\right)\right)$-in FIG. 6b. The singular value analysis captures the overall contribution of each nonlinear interaction term, thereby also verifying these findings.

Similarly in FIG.7a, we show the Lagrange multipliers obtained from MTEG analysis (Eq. (24) of the W parameters for $R=0.01$. Again it can be observed that the nonlinear terms $\delta w^{2}$ and $\gamma w u-\delta w v-$ from $\xi_{p_{2}}$ and $\xi_{p_{3}}$, respectively - are the dominant flow interactions contributing to MTEG in the WKH system. We obtain similar findings related to dominating flow interactions when comparing Lagrange multipliers obtained from the $R_{0}$ analysis results from Eq. 20. Waleffe discusses the importance of the nonlinearities $\delta w^{2}$ and $\gamma w u-\delta w v$ in feeding $\dot{v}$ and $\dot{w}$, thereby serving central roles in sustaining turbulence and conserving energy, respectively. We note that this analysis of Lagrange multipliers allowed the same dominant nonlinear flow physics to be identified without reliance upon any prior knowledge or physical insight. Similar trends are observed for other values of $R$. The same is true for the B\&T parameters.

When we investigate the Lagrange multipliers for the MTEG bound from Eq. (24), we observe that the Lagrange multiplier $\xi_{p 3}$ (shown in FIG 7a) associated with the nonlinear term $\gamma w u-\delta w v$ consistently increases in magnitude with increasing $R e$, while Lagrange multipliers $\xi_{p 0}, \xi_{p 1}$ and $\xi_{p 4}$ always have magnitude $\leq 1$. The multiplier $\xi_{p 3}$ has a magnitude of $\approx 110$ at $R e=150$ and it steadily increases by a factor of 6 at $R e=280$. It should be noted that this increase in magnitude of $\xi_{p_{3}}$ - while other multipliers are relatively constant - shows the dominance of the associated nonlinear term. The same can be verified by studying the maximum singular values, $\sigma_{\max }\left(\xi_{p_{i}} M_{i}\right)$ as shown in FIG. $7 \mathrm{~b}$.

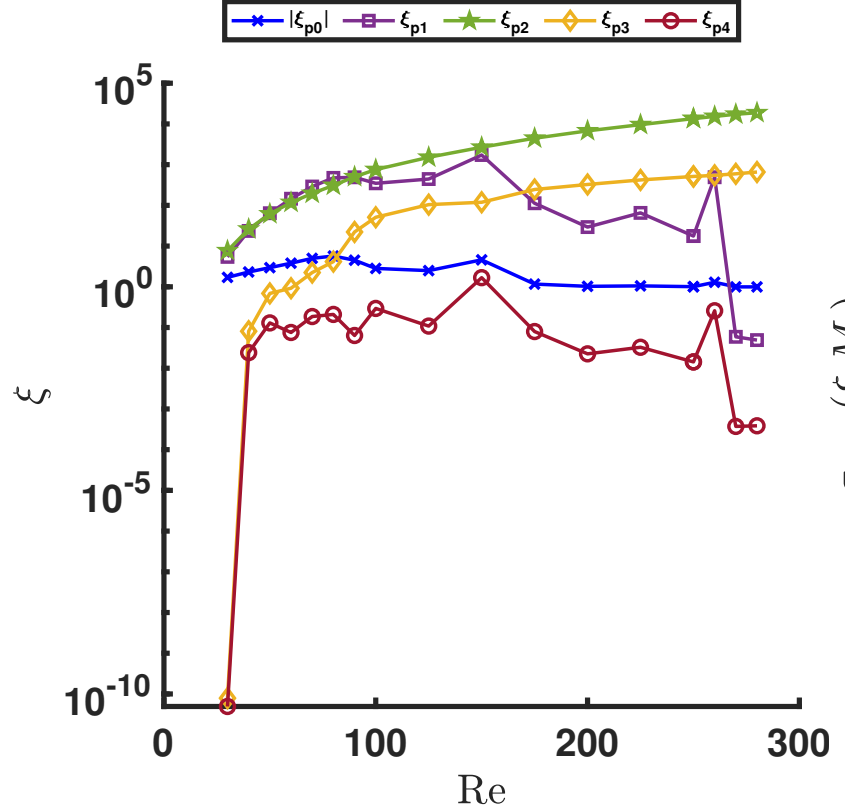

(a)

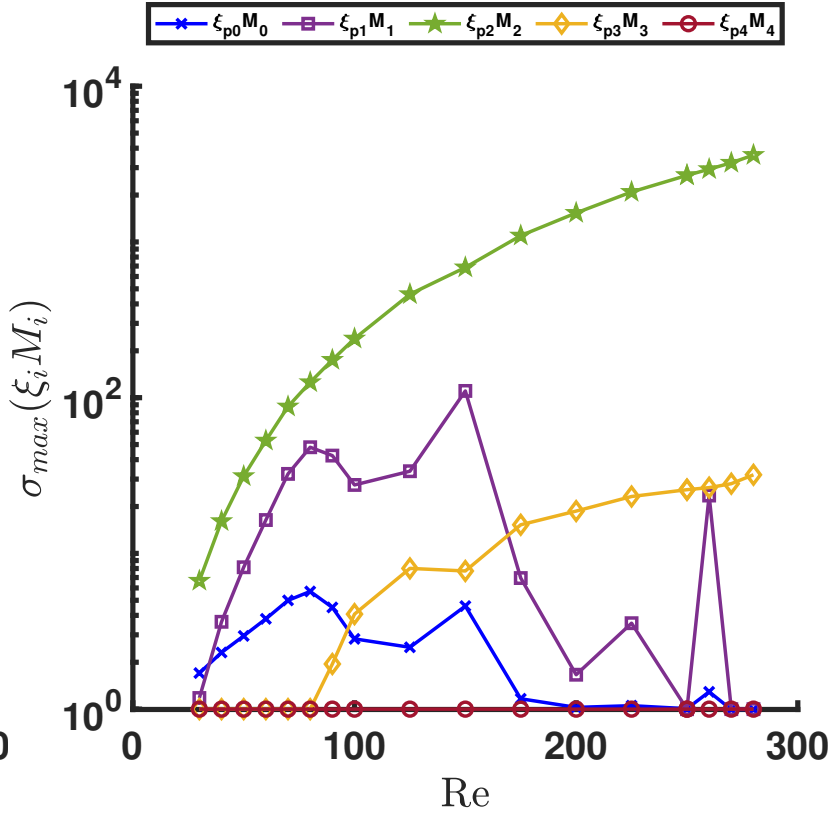

(b)

FIG. 6. Results obtained from solving $R_{0}$ in Eq. 20 with W parameters. The dominating nonlinear terms for stability can be identified by analyzing Lagrange multipliers $\xi_{p_{i}}$ in (a) and its associated singular values $\sigma_{\max }\left(\xi_{p_{i}} M_{i}\right)$ in (b).

To demonstrate the dominance of these nonlinear interactions, we perform MTEG analysis in Eq. 24 while retaining only the local constraints associated with the dominating nonlinear interactions $\left(\xi_{p_{2}}, \xi_{p_{3}}\right)$ and neglecting the other local interactions $\left(\xi_{p_{1}}, \xi_{p_{4}}\right)$. We choose $R=0.01$ as before, but now use only the lossless constraint along with constraints associated with $\xi_{p_{2}}$ and $\xi_{p_{3}}$ (see green line in FIG. 8) and compare results with the case where all the constraints are retained (see blue line in FIG. 8). The MTEG profile based on analysis using two dominating nonlinear interactions $\left(\delta w^{2}, \gamma w u-\delta w v\right)$ and the lossless constraint closely approximates the MTEG response of the whole nonlinear system. We observe similar qualitative trends for any other value of $R$ for which the optimization problem is feasible and also for the B\&T model parameters. 


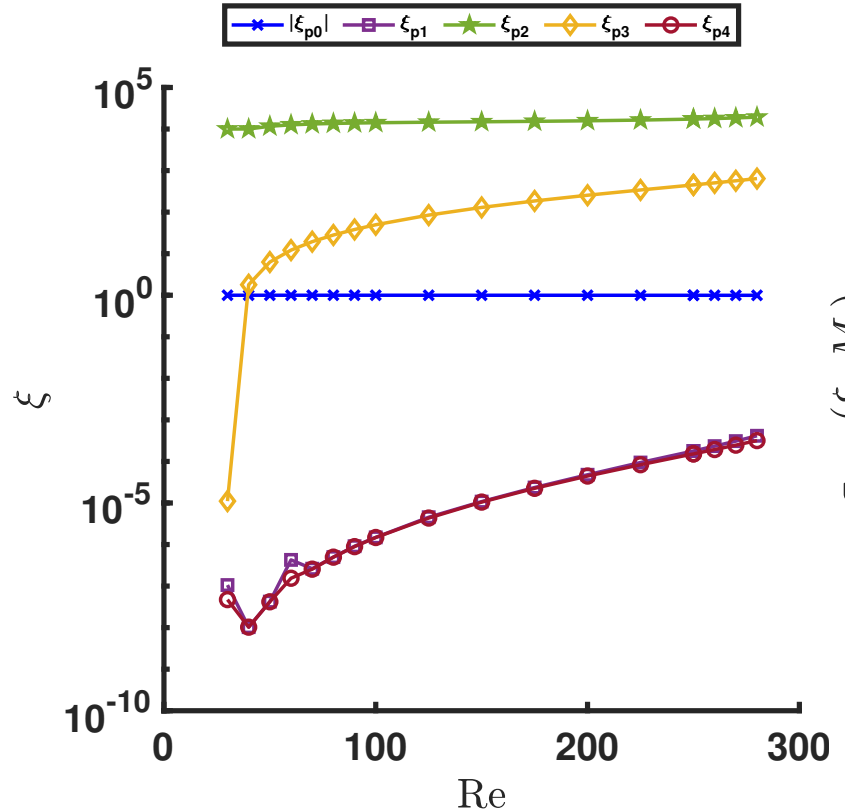

(a)

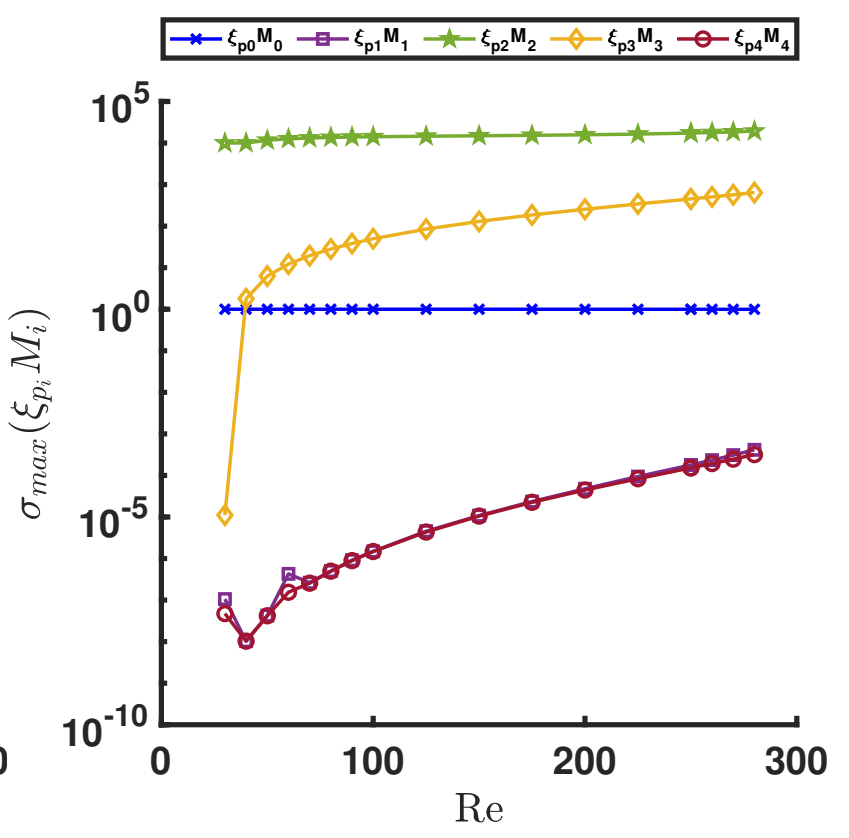

(b)

FIG. 7. Results obtained from solving for $q^{*}$ in Eq. (24). The dominant nonlinear terms for TEG can be identified by analyzing the dominant Lagrange multipliers $\xi_{p_{i}}$ in (a) and its associated singular values $\sigma_{\max }\left(\xi_{p_{i}} M_{i}\right)$ in (b).

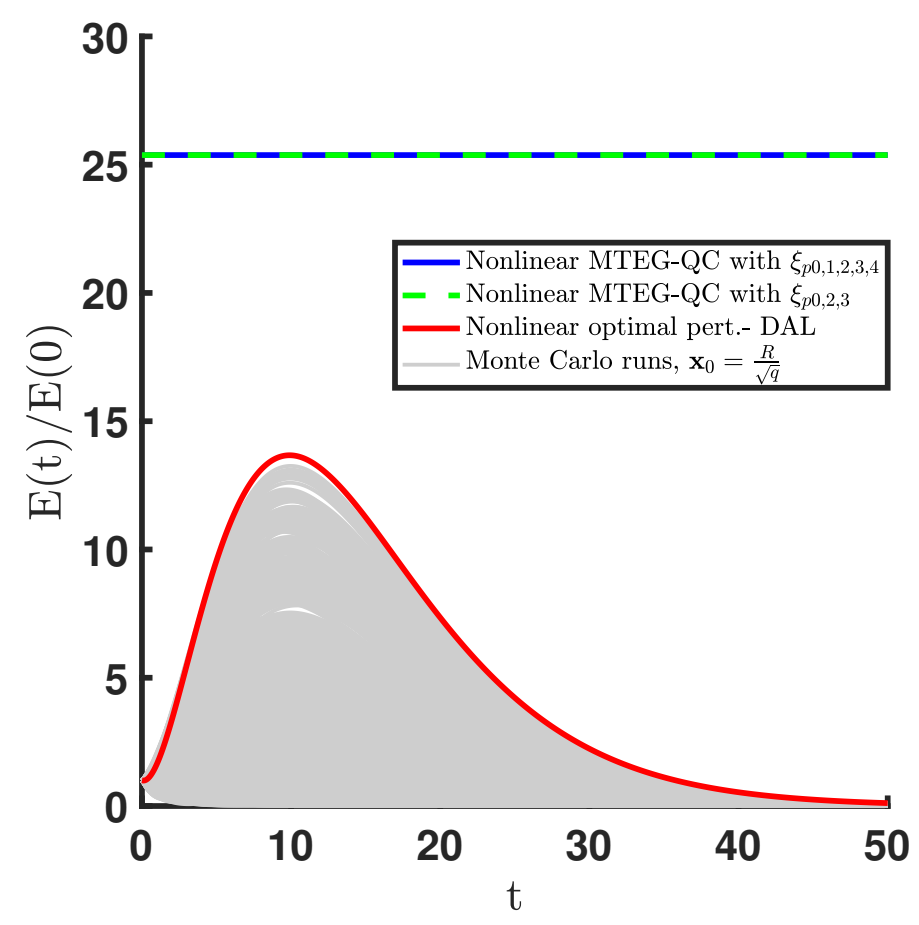

FIG. 8. Local MTEG analysis for W parameters with global lossless constraint and the two most dominant local constraints compared against the MTEG of system with global and all local constraints for $R e=100$ and $R=0.01$.

\section{CONCLUSION AND FUTURE WORK}

In this work, we presented a quadratic constraints framework to perform stability and transient energy growth analysis of nonlinear systems. The proposed framework facilitates stability and transient energy growth analysis in 
global and local settings around a given equilibrium point. The framework uses exact information from the linear dynamics, while nonlinear interactions are replaced by quadratic constraints that capture input-output properties of the nonlinearity.

We demonstrated the proposed analysis approach on the WKH model of transitional and turbulent flow. We first study the stability of the WKH model, for which the linear part is globally asymptotically stable for all Re. It is found that the nonlinear WKH system with W parameters is globally stable for $R e \leq 20$, consistent with previous results found in the literature. Similarly the global stability of the WKH system for B\&T parameters is verified for $R e \leq 2$. It is also observed that the energy conserving nonlinear terms destabilize the system beyond the globally stable regime.

In order to assess stability and maximum transient energy growth performance beyond the globally stable regime, we introduced a new "local" analysis framework to analyze local stability and transient energy growth properties. The local analysis provides an inner approximation for the region of attraction (ROA), which is determined by solving a GEVP. In addition to the ROA analysis, the solution procedure can be used to estimate permissible perturbation amplitudes. These analysis methods were compared with more computationally intensive SOS and DAL methods.

We also introduced a method for estimating maximum transient energy growth bounds when the system is either globally stable or simply locally stable. It was found that the maximum transient energy growth (MTEG) bound was unity below the critical Reynolds number for global stability. In the locally stable regime, we estimated the bounds on the MTEG and compared our results with those obtained from Monte Carlo simulations and DAL. Lastly, analyzing the Lagrange multipliers associated with each local constraint provided further insights into the physics. By comparing the relative magnitudes of the Lagrange multipliers, we were able to identify the dominating nonlinear interactions without any prior knowledge of the flow physics. The dominant nonlinear terms identified by this analysis were in agreement with the physical mechanisms originally described in [5].

The general QC framework proposed here shows promise in analyzing complex systems with quadratic and lossless nonlinearities, such as the incompressible NSE; however, there are computational challenges involved in generalizing this quadratic-constraint-based analysis for high dimensional fluids systems. The primary hurdle rests in the fact that computational demands of general purpose solvers for the convex optimization methods proposed here scale with $\mathcal{O}\left(n^{6}\right)$, where $n$ is the state dimension [42. Potential avenues forward may rely upon accurate reduced-order models, dedicated solvers, or some combination thereof. Nonetheless, the ideas proposed here establish exciting avenues for fluid flow analysis that - with further development - are expected to provide valuable insights about complex flow physics and their control.

\section{ACKNOWLEDGEMENTS}

This material is based upon work supported by the Air Force Office of Scientific Research under award number FA9550-19-1-0034 and the National Science Foundation under grant number CBET-1943988.

[1] Schmid, P. J. and Henningson, D. S., Stability and transition in shear flows, Springer, 2001.

[2] Schmid, P. J., "Nonmodal stability theory," Annual Review of Fluid Mechanics, Vol. 39, 2007, pp. 129-62.

[3] Trefethen, L. N., Trefethen, A. E., Reddy, S. C., and Driscoll, T. A., "Hydrodynamic stability without eigenvalues." Science (New York, N.Y.), Vol. 261, No. 5121, Jul 1993, pp. 578-84.

[4] Reddy, S. C. and Henningson, D. S., "Energy growth in viscous channel flows," Journal of Fluid Mechanics, Vol. 252, No. -1, Jul 1993, pp. 209.

[5] Waleffe, F., "Hydrodynamic stability and turbulence: beyond transients to a self-sustaining process," Studies in Applied Mathematics, 1995.

[6] Waleffe, F., "Transition in shear flows. Nonlinear normality versus non-normal linearity," Physics of Fluids, 1995.

[7] Sharma, A., Morrison, J, F., McKeon, B. J., and Limebeer, D.J.N. Koberg, W., "Relaminarisation of $R_{T}=100$ channel flow with globally stabilising linear feedback control," Physics of Fluids, 2011.

[8] Kerswell, R., "Nonlinear nonmodal stability theory," Annual Review of Fluid Mechanics, 2018.

[9] McKeon, B. J. and Sharma, A. S., "A critical-layer framework for turbulent pipe flow," Journal of Fluid Mechanics, 2010.

[10] Jovanovic, M. R. and Bamieh, B., "Componentwise energy amplification in channel flows," Journal of Fluid Mechanics, Vol. 534, 2005, pp. 145-183.

[11] Jovanovic, M. R., "From bypass transition to flow control and data-driven turbulence modeling: An input-output viewpoint," arXiv:2003.10104v1 [physics.flu-dyn], 2020.

[12] Khalil, H. K., Nonlinear systems, Prentice Hall, 2002.

[13] Taira, K., Brunton, S. L., Dawson, S. T. M., Rowley, C. W., Colonius, T., Mckeon, B. J., Schmidt, O. T., Gordeyev, S., Theofilis, V., and Ukeiley, L. S., "Modal analysis of fluid flows: an overview," AIAA Journal, 2017. 
[14] Taira, K., Hemati, M. S., Brunton, S. L., Sun, Y., Duraisamy, K., Bagheri, S., Dawson, S. T. M., and Yeh, C.-A., "Modal analysis of fluid flows: applications and outlook," AIAA Journal, Mar 2019.

[15] McKeon, B. J., "The engine behind (wall) turbulence: perspectives on scale interactions," Journal of Fluid Mechanics, Vol. 817, 2017.

[16] Sharma, A. S. and McKeon, B. J., "On coherent structure in wall turbulence," Journal of Fluid Mechanics, 2013.

[17] Sun, Y., Liu, Q., Cattafesta III, L. N., Ukeiley, L. S., and Taira, K., "Resolvent analysis of compressible laminar and turbulent cavity flows," AIAA Journal, 2019.

[18] Chavarin, A. and Luhar, M., "Resolvent analysis for turbulent channel flow with riblets," AIAA Journal, 2019.

[19] Heins, P. H., Jones, B. L., and Sharma, A. S., "Passivity-based output-feedback control of turbulent channel flow," Automatica, 2016.

[20] Damaren, C. J., "Laminar-turbulent transition control using passivity analysis of the orr-sommerfeld equation," Journal of Guidance, Control, and Dynamics, Vol. 39, No. 7, 2016, pp. 1602-1613.

[21] Damaren, C. J., "Transition control of the Blasius boundary layer using passivity," Aerospace Systems, Vol. 2, No. 1, 2018, pp. 21-31.

[22] Ahmadi, M., Valmorbida, G., Gayme, D., and Papachristodoulou, A., "A framework for input-output analysis of wallbounded shear flows," ArXiv ID 1802.04974v1, 2018.

[23] Joseph, D. D., Stability of fluid motions I, Springer-Verlag, New York, 1976.

[24] Goulart, P. J. and Chernyshenko, S., "Global stability analysis of fluid flows using sum-of- squares," Physica D, Vol. 241, 2012, pp. 692-704.

[25] Waleffe, F., Kim, J., and Hamilton, J., "On the origins of streaks in turbulent shear flows," Turbulent Shear Flows Springer, 1993.

[26] Henningson, D., "Comment on transition in shear flows. Nonlinear normality versus non-normal linearity," Physics of Fluids, 1996.

[27] Baggett, J. S. and Trefethen, L. N., "Low-dimensional models of subcritical transition to turbulence," Physics of Fluids, 1997.

[28] Prussing, J. E., "The principal minor test for semidefinite matrices," Journal of Guidance, Control, and Dynamics, Vol. 9, No. 1, 1986, pp. 121-122.

[29] Horn, R. A. and Johnson, C. R., Matrix analysis, Cambridge University Press, 1990.

[30] Gallier, J., "The Schur complement and symmetric positive semidefinite ( and Definite ) matrices," Complement, 2010.

[31] Whidborne, J. F. and McKernan, J., "On the minimization of maximum transient energy growth," IEEE Transactions on Automatic Control, Vol. 52, No. 9, Sep 2007, pp. 1762-1767.

[32] Grant, M. and Boyd, S., "CVX: Matlab software for disciplined convex programming, version 2.1," http://cvxr.com/cvx Mar 2014.

[33] Grant, M. and Boyd, S., "Graph implementations for nonsmooth convex programs," Recent Advances in Learning and Control, edited by V. Blondel, S. Boyd, and H. Kimura, Lecture Notes in Control and Information Sciences, SpringerVerlag Limited, 2008, pp. 95-110.

[34] ApS, M., The MOSEK optimization toolbox for MATLAB manual. Version 9.0., 2019.

[35] Boyd, S. and Ghaoui, L. E., "Method of centers for minimizing generalized eigenvalues," Linear Algebra and Applications, special issue on Linear Algebra in Systems and Control, 1993.

[36] Gahinet, P. and Nemirovskii, A., "General-purpose LMI solvers with benchmarks," Proceedings of the IEEE Conference on Decision and Control, 1993.

[37] Kerswell, R. R., Pringle, C. C., and Willis, A. P., "An optimization approach for analysing nonlinear stability with transition to turbulence in fluids as an exemplar," arXiv:1408.3539, 2014.

[38] Liu, C. and Gayme, D. F., "An input-output inspired method for permissible perturbation amplitude of transitional wall-bounded shear flows," arXiv:2006.16484, Jun 2020.

[39] Blanchini, F., "Set invariance in control," Automatica, Vol. 35, No. 11, 1999, pp. 1747 - 1767.

[40] Chen, C.-T., Linear System Theory and Design, CBS College publishing, 1984.

[41] Boyd, S., El Ghaoui, L., Feron, E., and Balakrishnan, V., Linear matrix inequalities in system and control Theory, Society for Industrial and Applied Mathematics, Jan 1994.

[42] Boyd, S. and Vandenberghe, L., Convex optimization, Cambridge University Press, 2004.

[43] Willems, J. C., "Dissipative dynamical systems part I: general theory," Archive for Rational Mechanics and Analysis, 1972. 


\section{Appendix A: Sector-bounded nonlinearities}

A comprehensive review of sector bounded systems can be obtained in $[12$, 43. Given a nonlinearity $\phi: \mathbb{R} \rightarrow \mathbb{R}$, $\phi$ lies in a sector $[\kappa, \beta]$ if for all $q \in \mathbb{R}, p=\phi(q)$ lies between the lines of slope $\kappa$ and $\beta$ at each point in time. This property can also written in terms of the input and output of the nonlinearity as a quadratic inequality of the form $(\beta q-\phi(q))(\phi(q)-\kappa q) \geq 0 \forall q \in \mathbb{R}$, or equivalently

$$
\begin{aligned}
{\left[\begin{array}{l}
q \\
p
\end{array}\right]^{T}\left[\begin{array}{cc}
-\kappa \beta & \frac{1}{2}(\kappa+\beta) \\
\frac{1}{2}(\kappa+\beta) & -1
\end{array}\right]\left[\begin{array}{l}
q \\
p
\end{array}\right] } & \geq 0 \forall q \in \mathbb{R}, \\
p & =\phi(q) .
\end{aligned}
$$

Graphically this is shown in FIG. 9, where the shaded region contains the nonlinearity $\phi$.

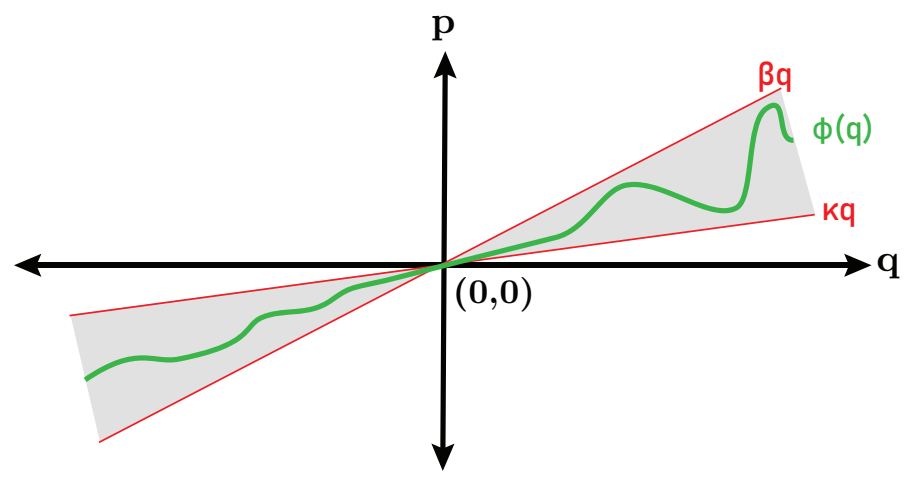

FIG. 9. Illustration of a generic sector bounded nonlinearity

\section{Appendix B: The set bounded by local region $R$ is invariant}

The role of $R$ in this analysis can be made more precise. Assume there is a feasible solution $P>0$ for the linear matrix inequality in Eq. 17). Then the Lyapunov function $V(\mathbf{x})=\mathbf{x}^{T} P \mathbf{x}$ satisfies $d V(\mathbf{x}(t)) / d t<0$ as long as $\mathbf{x}(t)^{T} \mathbf{x}(t) \leq R^{2}$. This implies that trajectories converge back to $\mathbf{x}_{e}=0$ if the initial conditions are sufficiently close to the origin. In particular, the constraint $P>I$ implies that $\mathbf{x}^{T} \mathbf{x}<V(\mathbf{x})$. A simple proof by contradiction can be used to demonstrate that if $V(\mathbf{x}(0))<R^{2}$ then: (i) the trajectory $\mathbf{x}(t)$ remains in the local region $\|\mathbf{x}(t)\|^{2} \leq R^{2}$ and (ii) the trajectory $\mathbf{x}(t)$ decays to the origin. In summary, the set $S_{R}:=\left\{\mathbf{x}: V(\mathbf{x})<R^{2}\right\}$ is a domain of attraction.

\section{Proof:}

Define the set $S_{R}:=\left\{\mathbf{x}: V(x)<R^{2}\right\}$. Assume $\mathbf{x}(0) \in S_{R}$ and let $\mathbf{x}(t)$ denote the corresponding state trajectory from this initial condition. Assume there exists a time $T_{1}$ such that $\mathbf{x}\left(T_{1}\right) \notin S_{R}$ and let $T_{0}$ be the smallest (infimum) of times such that $\mathbf{x}(t) \notin S_{R}$. The solution $\mathbf{x}(t)$ is a continuous function of time and hence $\mathbf{x}(t) \in S_{R}$ for all $t \in\left[0, T_{0}\right)$ and, moreover, $\mathbf{x}(t)$ is on the boundary of $S_{R}$ so that $V\left(\mathbf{x}\left(T_{0}\right)\right)=R^{2}$. As noted above, $P>I$ implies that if $\mathbf{x}(t) \in S_{R}$ then $\|\mathbf{x}(t)\|^{2}<R^{2}$. Therefore, the local quadratic constraints are valid for all $t \in\left[0, T_{0}\right]$. The constraints in Eq. (17) imply that, for a sufficiently small $\epsilon>0$, the Lyapunov function satisfies $d V(\mathbf{x}(t)) / d t \leq-\epsilon \mathbf{x}(t)^{T} \mathbf{x}(t) \forall t \in\left[0, T_{0}\right]$. Integrating yields the following bound for any $\mathbf{x}(0) \neq 0$ :

$$
V\left(\mathbf{x}\left(T_{0}\right)\right) \leq V(\mathbf{x}(0))<R^{2}
$$

This contradicts the assumption that $V\left(\mathbf{x}\left(T_{0}\right)\right)=R^{2}$ and hence trajectories must remain in $S_{R}$. Moreover, the Lyapunov condition $d V / d t \leq-\epsilon \mathbf{x}(t)^{T} \mathbf{x}(t) \forall t \in\left[0, T_{0}\right]$ implies that the trajectories in this region decay asymptotically back to the origin. 